\title{
Itinéraires Itinéraires
}

Littérature, textes, cultures

\section{Charlot, un clown raconte le mélodrame}

\section{Anne Poggioli}

\section{(2) OpenEdition}

\section{Journals}

Édition électronique

URL : http://journals.openedition.org/itineraires/2442

DOI : $10.4000 /$ itineraires. 2442

ISSN : 2427-920X

Éditeur

Pléiade

\section{Référence électronique}

Anne Poggioli, «Charlot, un clown raconte le mélodrame », Itinéraires [En ligne], 2014-2 | 2015, mis en ligne le 17 juillet 2015, consulté le 02 octobre 2020. URL : http://journals.openedition.org/itineraires/ 2442 ; DOI : https://doi.org/10.4000/itineraires.2442

Ce document a été généré automatiquement le 2 octobre 2020.

\section{(c) (1) (9)}

Itinéraires est mis à disposition selon les termes de la licence Creative Commons Attribution - Pas d'Utilisation Commerciale - Pas de Modification 4.0 International. 


\title{
Charlot, un clown raconte le mélodrame
}

\author{
Anne Poggioli
}

En toute innocence, alors que je reprenais le refrain, j'imitai la voix de ma mère qui se brisait et je fus surpris de voir quel effet cela avait sur

l'auditoire.

Il y eut des rires et des acclamations, et une nouvelle pluie de monnaie.

[...] Ce soir-là marqua ma première apparition sur la scène et la dernière de ma mère. Charles Chaplin (1978: 19)

Dans son autobiographie, Histoire de ma vie, Chaplin raconte sa première expérience de la scène à l'âge de cinq ans : il doit en urgence remplacer sa mère, artiste de music-hall, atteinte d'une extinction de voix en pleine représentation. "En toute innocence", l'enfant imite la voix cassée de sa mère et déclenche, à sa grande surprise, les rires du public. Un clown est né. Chaplin insiste sur l'innocence de l'enfant qu'il était et sur sa surprise : c'est ce sentiment qu'il gardera dans sa mémoire en créant le personnage de Charlot ; et c'est l'une des clefs pour accéder à l'art du clown. Du surgissement spontané de ce rire, il tirera les leçons et percevra la puissance de la parodie qui, loin d'être une caricature outrancière et cynique, est la citation décalée d'une œuvre.

Le music-hall, extrêmement répandu et apprécié au XIX siècle, en Angleterre et aux États-Unis, est d'une inépuisable variété. Keaton et Chaplin ont tous deux fait leurs premières armes sur les scènes de cet art populaire. $Y$ convergent alors presque toutes les formes de spectacle que le music-hall s'approprie avec avidité, cite et déforme. Très jeune, Charlie Chaplin se produit dans les salles les plus prestigieuses de son pays, sillonnant l'Angleterre lors de longues tournées. Parmi les numéros les plus prisés du public, figuraient les parodies de mélodrames qui avaient connu un franc succès durant la saison théâtrale. Le public aimait reconnaitre les codes et les thèmes de ces mélodrames transcrits sur un mode parodique. Dans ses films, sans cesse nourris de 
références au mélodrame, Chaplin ne s'approprie-t-il pas l'art de la parodie en y insufflant la puissance émotionnelle du clown? S'il n'est ni le premier ni le seul à son époque à introduire les conventions du mélodrame au cinéma ${ }^{1}$, ne fait-il pas œuvre originale lorsqu'il convie devant sa caméra, pour la plus cathartique des rencontres, les canevas du mélodrame et la figure du clown? Le clown est appliqué, d'une absolue bonne foi, et cet excès d'application crée un hiatus entre ce qu'il s'attache à reproduire et sa propre identité, décalée et précaire. Il est le corps étranger qui permet de faire dériver la citation vers la parodie. Chaplin utilise les codes du jeu mélodramatique, les amplifie, les cadence, les détourne. Le cinéaste provoque ainsi une intrusion dans le tissu de la fable, en y créant défauts et syncopes jusqu'à rester en équilibre entre burlesque et pathétique, ce qui suscite infailliblement l'émotion complice des spectateurs. Avec Chaplin, le mélodrame qui faisait pleurer Margot - selon le célèbre vers de Musset - fait rire le public.

3 En faisant entrer à la fois le cirque et les codes du mélodrame dans le dispositif cinématographique, Chaplin invente une grammaire burlesque très particulière. La collision entre ces deux univers, entre ces deux styles de jeu, permet au cinéaste de procéder à une convulsion de la forme et à une dislocation de la fable. L'interprétation de Charlot par Chaplin relève en effet du jeu masqué ${ }^{2}$ le clown se rêve en héros de mélodrame.

\section{Le jeu masqué de Chaplin}

On a coutume de dire que le clown burlesque, que ce soit « The Tramp ${ }^{3}$ », le personnage de Keaton ou Monsieur Hulot de Tati, a enlevé son nez rouge pour sortir de la piste du cirque et aller dans la rue, grâce au cinéma. Mais, s'il ne porte plus son nez, le clown burlesque au cinéma reste un masque : il a en quelque sorte avalé son nez rouge. Son corps entier, sa cadence, son caractère inadapté aux réalités sociales et à leurs codes de comportement, son innocence, signent son appartenance à la famille des masques. Tous les codes du jeu masqué, son solfège, élaborés en premier lieu au tournant des XIX et $\mathrm{Xx}^{\mathrm{e}}$ siècles par les quatre maîtres russes ${ }^{4}$-Stanislavski, Meyerhold, Vakhtangov et Mikhail Tchekhov - se retrouvent dans la composition de l'art de Chaplin. Paradoxalement, ce dernier se trouve être à la fois l'héritier et le générateur de cette approche radicalement différente de l'interprétation.

5 En effet, le personnage de Charlot s'inscrit dans la lignée du type comique populaire qui relie le théâtre de foire, la commedia dell'arte, le music-hall et le cirque. Toutes ces formes spectaculaires représentent pour les avant-gardistes ${ }^{5}$ ce qu'ils nomment la théâtralité authentique ${ }^{6}$, source à laquelle l'acteur $\mathrm{du} \mathrm{xx}^{\mathrm{e}}$ siècle, entrant dans la modernité, doit puiser. Ce "pantin sentimental»-c'est ainsi que Chaplin nommait Charlot - est également le contemporain de tous les penseurs et artistes du mouvement, de François Delsarte à Émile Jaques-Dalcroze, d'Isadora Duncan à Oskar Schlemmer. Il est ainsi la synthèse de la pantomime ancestrale et du tracé graphique né de la révolution industrielle ${ }^{7}$.

D'autre part, Charlot représente un modèle pour nombre d'avant-gardistes et se trouve à la source de la réflexion de deux grands penseurs du théâtre : Meyerhold et Brecht.

On sait que Meyerhold, fort de ses expériences cinématographiques en tant que réalisateur et acteur, va repenser le théâtre et enrichir sa quête d'un jeu fondé sur la 
convention. Dans La Forêt d'Ostrovski, son acteur Igor Ilinski conjugue savamment un jeu théâtral empruntant à la fois à Arlequin, à la figure du gracioso espagnol, au baladin russe, au clown de cirque et au jeu cinématographique, car Meyerhold le dirige en s'inspirant du personnage de Charlot. Pour le metteur en scène russe, le cinéma burlesque a remplacé le vide laissé par l'absence du théâtre de foire: «Le cinématographe nous rapproche du théâtre de foire, du cirque et des variétés » (PiconVallin 1990 : 233). Ainsi, pendant qu'Eisenstein, son élève, annonce haut et fort la mort du théâtre et commence sa carrière de cinéaste, Meyerhold travaille à la régénération du théâtre en empruntant et transcrivant des éléments du jeu cinématographique pour consolider le langage théâtral. Sa célèbre biomécanique, définie en 1918 et enseignée à partir des années 1920, s'inspire ainsi autant de traditions anciennes et populaires occidentales et orientales, de pensées nouvelles comme la réflexologie de Pavlov, le taylorisme et la sur-marionnette de Gordon Craig que de l'exemple de Chaplin.

Pour Brecht, Charles Chaplin est l'acteur idéal. Il admire chez lui l'absence de tout psychologisme ${ }^{8}$ car en aucun cas l'interprète ne subordonne le signe au sens. Ainsi, lorsqu'il clame son admiration pour Karl Valentin, autre grand clown, Brecht précise que c'est parce que, comme dans la pantomime de Chaplin, son art choisit «[...] la renonciation presque complète aux jeux de physionomie et à la psychologie de pacotille» (Brecht 1972 : 58). L'interprétation de l'acteur n'illustre pas la fable, elle la dédouble, lui donne une épaisseur. Brecht apprécie fort la gestuelle de Chaplin, qui use sans cesse de la citation et produit ainsi la distanciation. Cet acteur représente aussi pour le théoricien un modèle sur le plan politique. Comme Roland Barthes l'a montré dans Mythologies, l'acteur anglais et le metteur en scène allemand ont en commun d'être les seuls à avoir représenté « l'homme à la veille de la Révolution, c'est-à-dire l'homme seul, encore aveugle [...]» (Barthes $1957: 39$ ) et « au-dessous de la prise de conscience politique » (Ibid. : 40). Les personnages de Brecht ressemblent à Charlot qui

[...] montre sa cécité au public de telle sorte que le public voit à la fois l'aveugle et son spectacle; voir quelqu'un ne pas voir, c'est la meilleure façon de voir intensément ce qu'il ne voit pas : ainsi au Guignol, ce sont les enfants qui dénoncent à Guignol ce qu'il feint de ne pas voir (Barthes 1957 : 39).

9 Ainsi, Chaplin offre à Brecht un modèle idéal pour ses théories théâtrales ${ }^{9}$ - et notamment pour l'élaboration de l'acteur épique et du Verfremdungseffekt.

Comme chez l'acteur nouveau, désiré par les avant-gardistes, tout le corps de Chaplin, dans sa globalité, est engagé dans le processus de jeu, de façon permanente, suivant un flux continu, même dans les moments d'immobilité. Chaplin raconte le monde et en transcrit sa vision sous une forme graphique précise, mécanique, lisible par tous. L'acteur ne fusionne pas avec son personnage, il le donne à voir, le raconte. Le personnage n'a aucune existence autonome qui préexisterait à celle de l'acteur : c'est un masque ${ }^{10}$ et l'acteur est donc en adresse constante au public même quand le jeu n'est pas frontal et que l'interprète ne regarde pas les spectateurs ou la caméra. Dans la tradition masquée, les acteurs s'adressent aux spectateurs et avouent ainsi la représentation. Chaplin s'inspire de cet héritage, affirme cette nécessité qui, transposée au cinéma, prend des allures d'audacieuse provocation. Charlot est avide de l'autre, il le prend à témoin, le provoque, lui confie son égarement, lui offre en partage sa maladresse, son désir de bien faire se heurtant à l'échec. Le public, ainsi pris à partie, est invité à déchiffrer la fable. Quand le clown surgit dans le champ du mélodrame, l'art du contrepoint et de l'irruption, fondement de son interprétation, éveille constamment la conscience critique du spectateur. 
11 Déjà, dans la première apparition du personnage de Charlot au cinéma, en 1914, le jeu est frontal. Kids auto race, court-métrage, raconte une course automobile pour enfants. Dans le cadre, le public de la course est présent ainsi qu'une équipe de tournage installée pour filmer l'événement. Or, Charlot vient perturber le tournage, il se pose dans le champ de la caméra, s'impose, faisant obstacle à la fois au tournage prévu dans la fiction et au film lui-même. On lui demande de partir, on le pousse assez violemment car il affiche une incompréhension totale de la situation, prend à témoin le spectateur du film par l'usage répété de regards caméra. Il a en quelque sorte un pied dans la fable et un pied dans la salle de projection. L'objectif de la caméra filmant l'ensemble se confond quelquefois avec celui de la caméra de la fable. Charlot les affronte, les confond, dénonce l'illusion, avoue le film et avoue son jeu. Il se sait regardé, il se sait filmé et le raconte aux spectateurs. Dès le premier film de Charlot, le dispositif est donc clairement posé.

Fig. 1. Extraits de Kids Auto Race (1914)

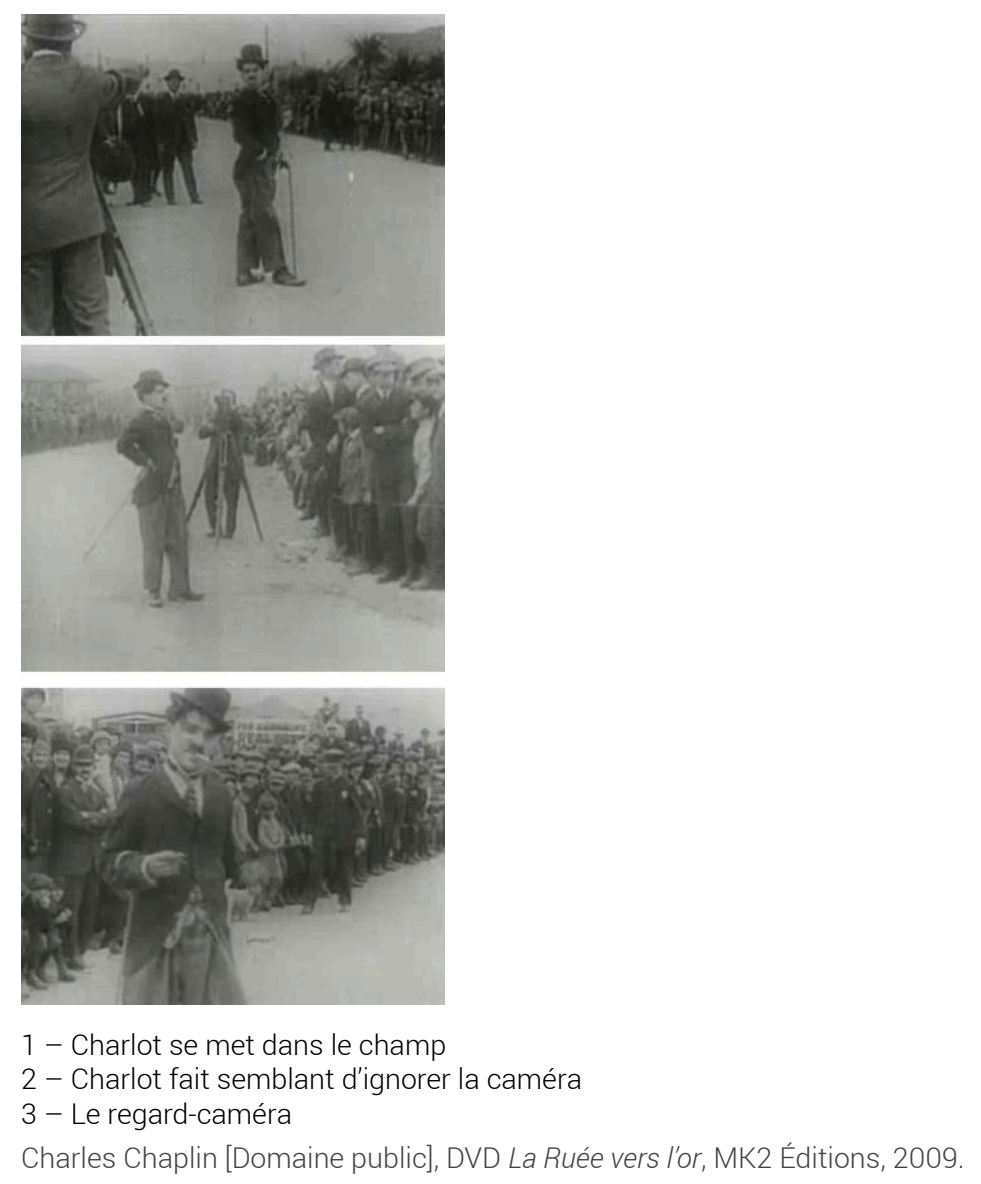

\section{La parodie du mélodrame dans La Ruée vers l'or}

\section{Le « démontage » du réalisme et du naturalisme}

The Gold Rush (La Ruée vers l'or) sort en 1925. Le film commence à la façon d'un documentaire sur les chercheurs d'or. Le cadre se veut réaliste, des hommes en longue file indienne gravissent des montagnes enneigées. Puis Charlot apparaît dans le cadre 
en produisant la fausse note nécessaire : il porte son indispensable costume de dandy vagabond, en opposition criante avec le contexte. Avec sa canne, son chapeau melon trop étroit et sa célèbre démarche due à des chaussures inadaptées à son pied, le long d'un sentier très étroit et escarpé, il introduit la dissonance et procède à un véritable «démontage » du réalisme. Le rire du spectateur jaillit, évidemment: on s'attend au pire.

Fig. 2. Extraits de The Gold Rush (1925)
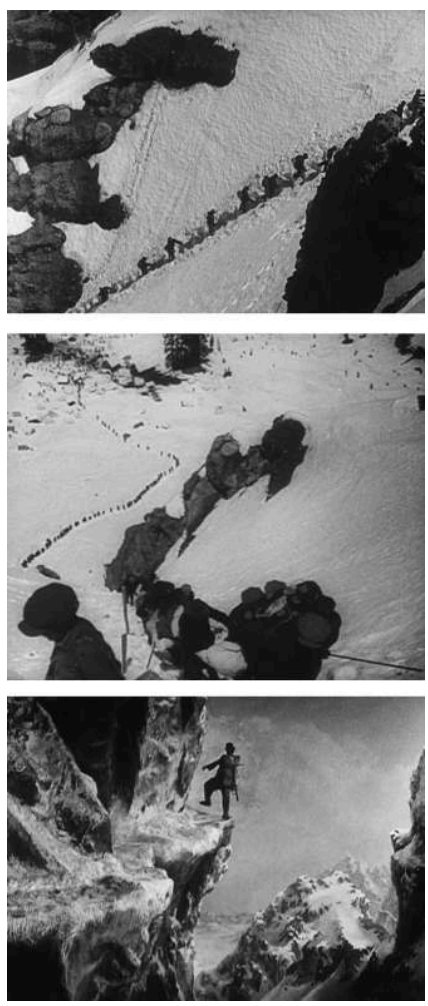

1 et 2 - Les chercheurs d'or filmés dans un cadre réaliste

3 - Charlot fait irruption dans ce cadre

Charles Chaplin [Domaine public], DVD La Ruée vers l'or, MK2 Éditions, 2009.

Plus tard, lors de la célèbre séquence de la cabane en équilibre au-dessus du vide, c'est métaphoriquement le naturalisme que Chaplin fait chavirer. La bicoque, pauvrement équipée, isolée dans la montagne, est prise dans la tourmente d'une nature déchaînée. Voilà un décor idéal pour un drame naturaliste exaltant des sentiments de compassion. Mais dans cette bicoque, le clown va bousculer, malgré lui, le scénario - qu'il ignore. Il introduit le désordre, nous entraîne dans une comédie et lorsque la cabane se retrouve au fond d'un ravin, c'est le drame qui est mis à mort par Chaplin. 


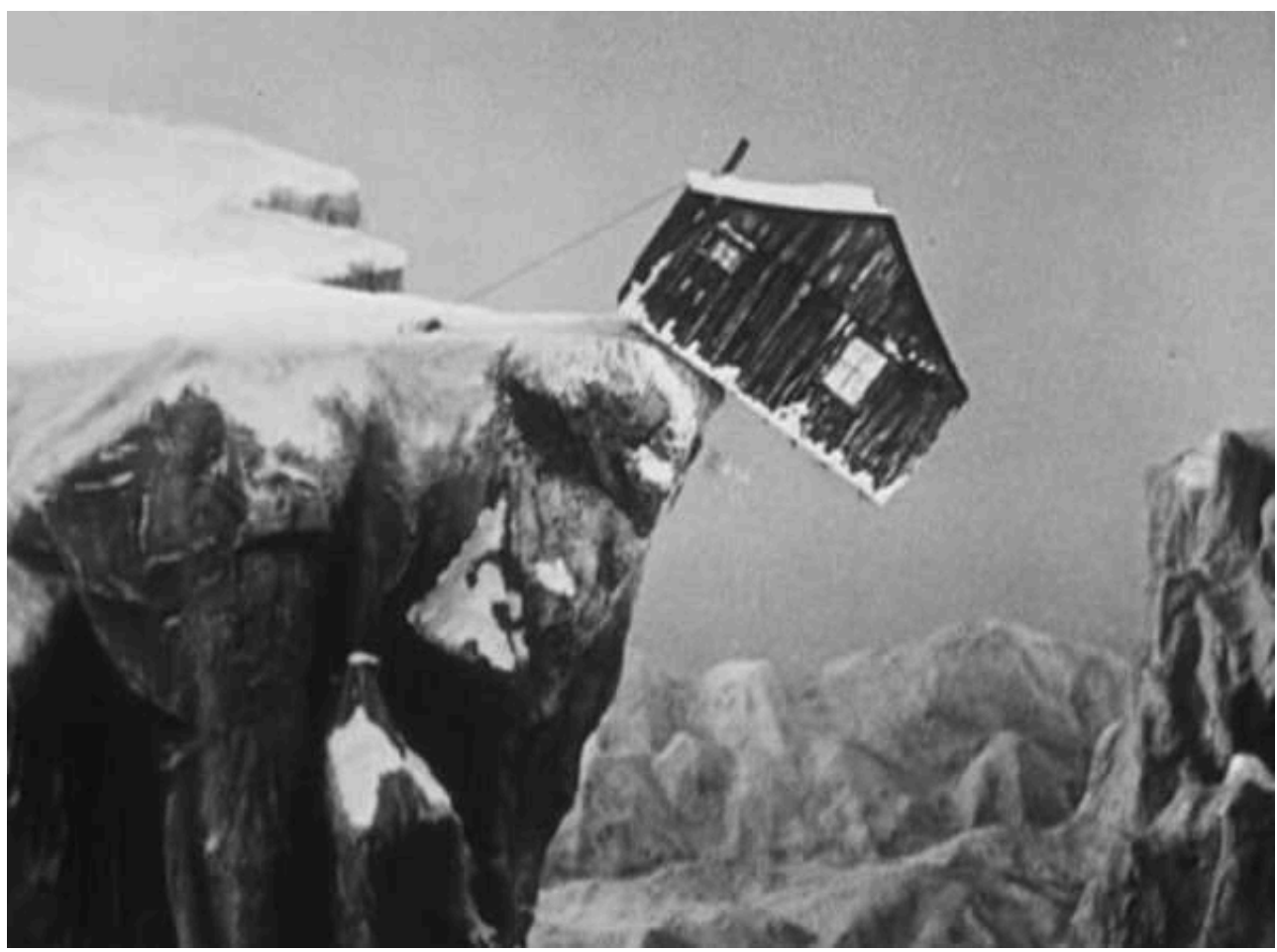

Mise à mort de la bicoque naturaliste

Charles Chaplin [Domaine public], DVD La Ruée vers l'or, MK2 Éditions, 2009.

\section{Chaplin cite le jeu mélodramatique}

Le mélodrame au xix ${ }^{e}$ siècle, excroissance du drame bourgeois du siècle précédent ${ }^{11}$, se caractérise par l'exagération des effets et l'outrance du jeu. Chaplin cite la gestuelle mélodramatique et, en la citant, la pousse à bout et subvertit l'ordre conventionnel du genre. Cette discordance produit un effet d'étrangeté - pour reprendre un terme brechtien ${ }^{12}-$, un hiatus entre la référence et son interprétation en forme de dérive. Prenons pour exemple, à la suite de James Naremore dans Acting in the Cinema, la scène des adieux de Charlot à Georgia, la femme qu'il aime. Dans le cabaret où Georgia travaille, Charlot retrouve son ami Big Jim qui lui demande de l'accompagner jusqu'à la mine d'or qu'il vient de découvrir. Il lui promet de faire sa fortune. Charlot décide de partir et doit donc faire ses adieux à la danseuse. L'acteur Chaplin utilise alors toute la panoplie de l'amour courtois ${ }^{13}$. Tel un héros tout droit sorti d'un film de cape et d'épée, il grimpe et saute avec agilité jusqu'au premier étage du cabaret, comme pour délivrer sa belle. Aucun obstacle ne semble pouvoir l'empêcher d'aller lui baiser la main. Puis, il décline toute la gestuelle du genre. Il jure à Georgia un amour éternel en portant la main à son cœur, il se hisse sur la pointe des pieds, tend le bras et adopte une posture hérö̈que - digne des dessins caricaturant les recherches de Delsarte ${ }^{14}$ qui circulaient aux États-Unis - en annonçant son départ en quête de fortune.

Ces gestes sont repérables, identifiés par le spectateur comme faisant référence à un jeu théâtral emphatique d'usage dans les mélodrames du XIX siècle et dans un certain cinéma muet du tout début $\mathrm{du} \mathrm{xx}^{\mathrm{e}}$ siècle. Chaplin procède donc par référence et légende ce que son personnage ressent. Charlot le clown s'est trompé de scène, il se 
croit au théâtre et il est au cinéma, il se prend pour un héros qui avant d'affronter force dangers jure un amour éternel à sa promise alors qu'il n'est qu'un petit bonhomme ridicule. La réalité se présente d'ailleurs à lui sous la forme du bras puissant de Big Jim qui le sort littéralement du cadre et l'oblige à quitter ce rôle pour l'entraîner vers un autre. Charlot accepte, se soumet, et mettra la même conviction à trouver sa place dans le scénario de Big Jim.

Fig. 4. Extraits de The Gold Rush (1925)
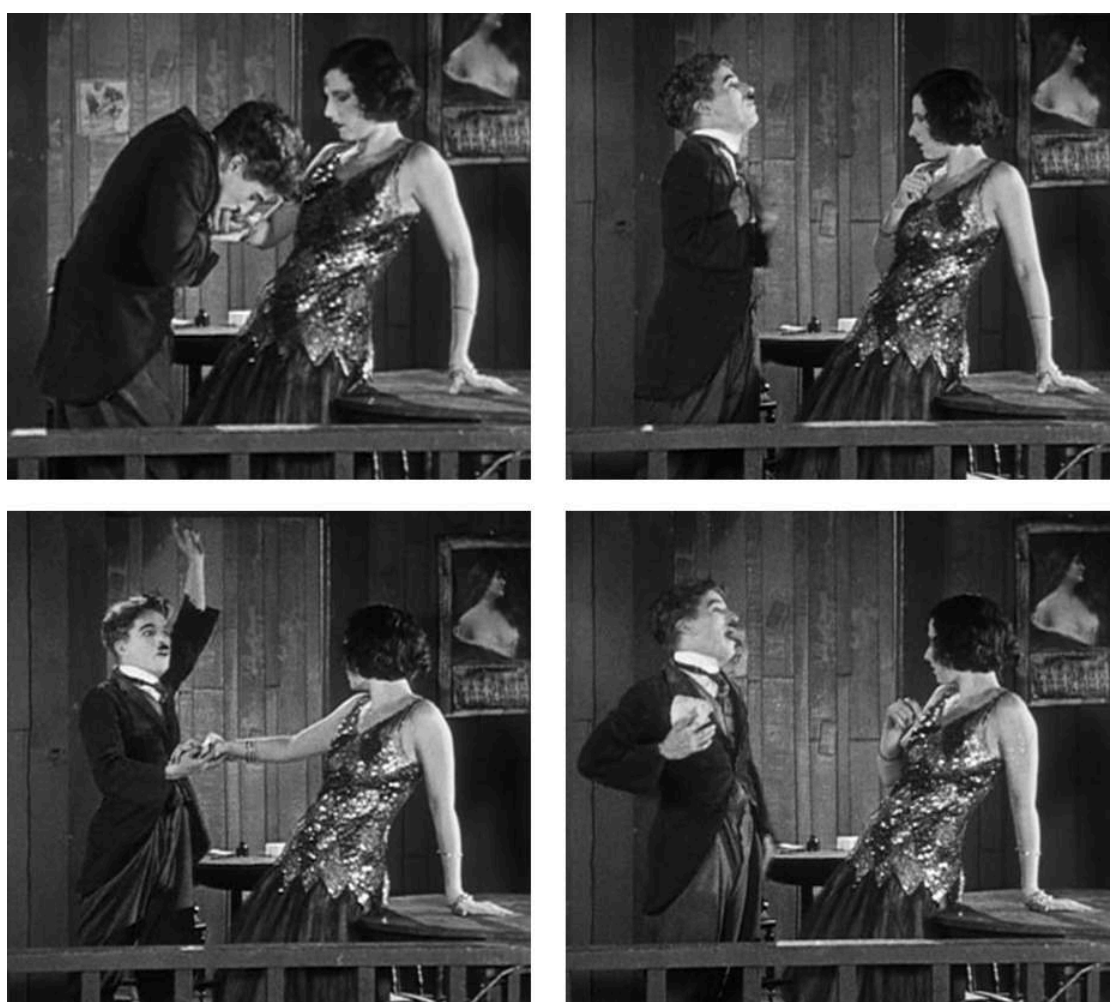

1 - Les adieux de Charlot à Georgia : le baise-main

2 - L'acteur exagère des postures théâtrales bien connues de tous, comme la main sur le cœur

3 - Chaplin pousse à l'extrême le geste mélodramatique

4 - Le drame bascule dans la parodie et la comédie

Charles Chaplin [Domaine public], DVD La Ruée vers I'or, MK2 Éditions, 2009.

C'est un clown, l'innocence et la foi se heurtant à l'incapacité d'être ce qu'il rêverait d'être. Son costume traduit ce décalage entre son origine humble et pauvre et son aspiration à paraître un homme du monde qui a sa place dans la société : le chapeau melon et la veste sont trop étroits, le pantalon et les chaussures sont trop grands : rien ne va. Le clown rêve d'être un jeune premier, et comme le précise James Naremore, il en rêve avec une passion folle et un engagement de tout son être : «[...] he is a tramp foolishly behaving like a lover ${ }^{15} »$ (Naremore $\left.1988: 122\right)$. De ce décalage fondamental du clown avec la réalité qui l'entoure, naît le rire. L'œil de la caméra, sensible au moindre battement de cils de l'acteur, amplifie la dimension du jeu. Le geste mélodramatique est poussé à l'extrême par l'acteur qui l'arrache ainsi au drame pour le faire dériver, le précipiter, dans la comédie. En accentuant ses mouvements jusqu'à la limite du possible, en en dévoilant le mécanisme, Chaplin franchit la limite entre drame et comédie burlesque. L'acteur lui-même nous a laissé de nombreux témoignages sur 
l'exigence et la précision de son interprétation et la relation complice qu'il cherche à établir avec le spectateur :

Je me suis spécialisé dans un type de comédie: la pantomime. Je l'ai mesurée, calibrée, étudiée. J'ai réussi à établir les principes exacts qui gouvernent son action sur le public. Un certain rythme, un certain tempo. (Cité dans Robinson 1995 :121)

Voilà des propos dignes d'un biomécanicien de Meyerhold. Chaplin contrôle parfaitement ce que Naremore qualifie de «[...] comic exageration of old-fashioned, stagy postures ${ }^{16}$ " (Naremore 1988: 122). Comme le dit Roland Barthes à propos de l'acteur épique brechtien, « [...] le sens est mis dans le geste et dans la coordination des gestes » (Barthes [1973] 2002 : 343). Cette expression qualifie à la perfection le jeu de l'acteur. "Mes pieds sont-ils dans le champ ? Ils sont aussi importants que ma tête", répétait sans cesse le cinéaste à Robert Florey, son assistant sur le tournage de Monsieur Verdoux.

Chaplin invite le cirque dans le mélodrame et sollicite la vivacité du spectateur pour éveiller son regard critique sur les mécanismes des fables bourgeoises.

\section{Chaplin fait exploser le mélodrame dans City Lights}

Chaplin introduit dans le cadre du mélodrame le clown Charlot qui, telle une mine posée à dessein, ne pourra que provoquer l'explosion de ce cadre.

\section{La lutte du cirque et du mélodrame}

Dans City Lights (Les Lumières de la ville), en 1931, le public assiste au début du film au sauvetage du mélodrame par la comédie. Lorsque Charlot rencontre le millionnaire excentrique, tous les codes du mélodrame sont posés.

À son entrée dans le champ, l'ombre du millionnaire projetée sur le mur évoque un film expressionniste. C'est la nuit. Le personnage porte un costume de soirée, attribut incontournable du rôle. Il fait son entrée par l'escalier et une fois arrivé au bord de l'eau, se met à manipuler tous les accessoires qui mettent en évidence son intention de se suicider. Ce sont des accessoires de mélodrame, de Grand Guignol : la corde, la pierre, nécessaires à la scène typique du mari abandonné, désespéré, voulant se donner la mort. L'acteur Harry Myers emprunte son style de jeu, très codifié, au mélodrame le plus outrancier : regards au ciel et sourcils levés.

Lorsque « The Tramp » fait son entrée, son jeu est très simple, presque neutre, mais son apparence dénonce immédiatement l'opposition de style avec son partenaire. Progressivement, Charlot, aspiré par le mélodrame, va cependant fusionner avec lui. Quand il comprend les intentions du suicidaire, il se met au diapason et utilise toute la gestuelle delsartienne. Mais le clown réapparaît au moment où Charlot veut signifier que la vie vaut la peine d'être vécue et que "demain sera un autre jour ", selon le conseil de rigueur dans ce genre de situation. Il se produit en effet un premier accident, une première syncope dans la fable: quand Charlot cherche à arracher des mains du suicidaire la pierre attachée à la corde prévue pour la noyade, elle lui tombe sur le pied. Quand, dans un élan lyrique, il se frappe la poitrine, le voilà secoué d'une quinte de toux; sa frêle constitution n'est décidément pas celle d'un héros. Sans cesse, les élans passionnés du clown provoquent sa chute. 
Fig. 5. Extraits de City Lights (1931)
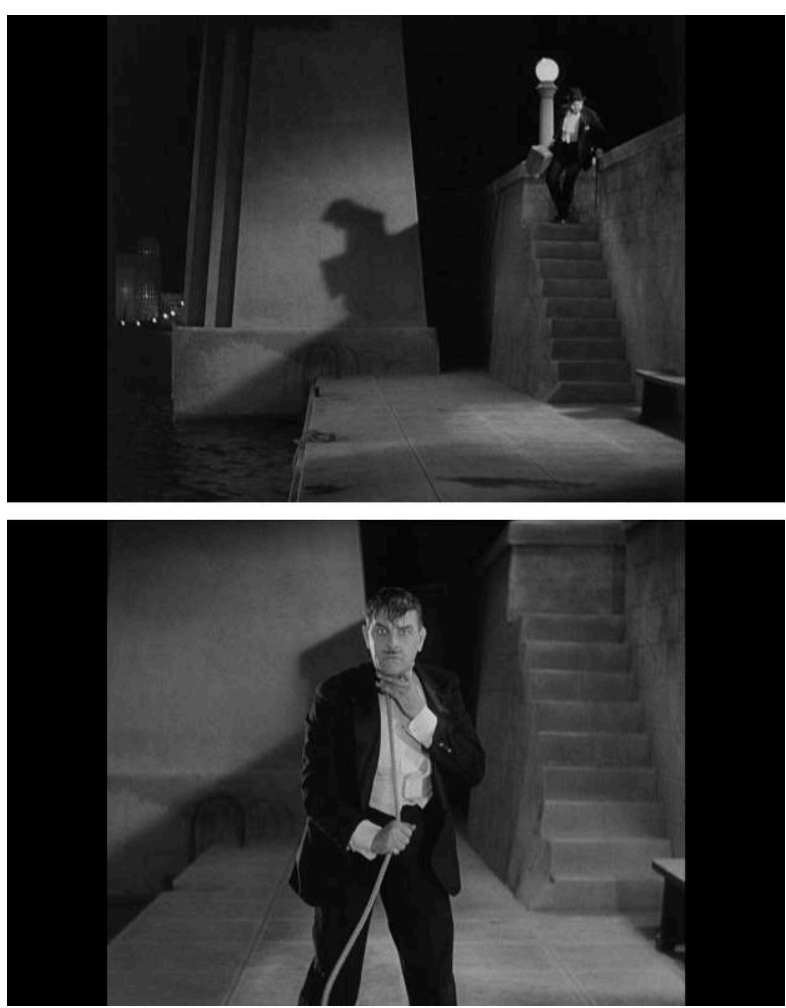

1 - Un décor de drame expressionniste (11'42)

2 - Le suicidaire se passe la corde au cou (11'59)

Charles Chaplin [Domaine public], DVD Les Lumières de la ville, MK2 Éditions, 2008.

Son application aussi grande que sa maladresse font basculer le mélodrame dans la comédie et les deux personnages finissent par tomber à l'eau. La noyade n'a rien de tragique, elle est burlesque et vouée à l'échec: c'est le mélodrame qui tombe à l'eau dans un plongeon démystifiant tout héroïsme. 
Fig. 6. Extraits de City Lights (1931)
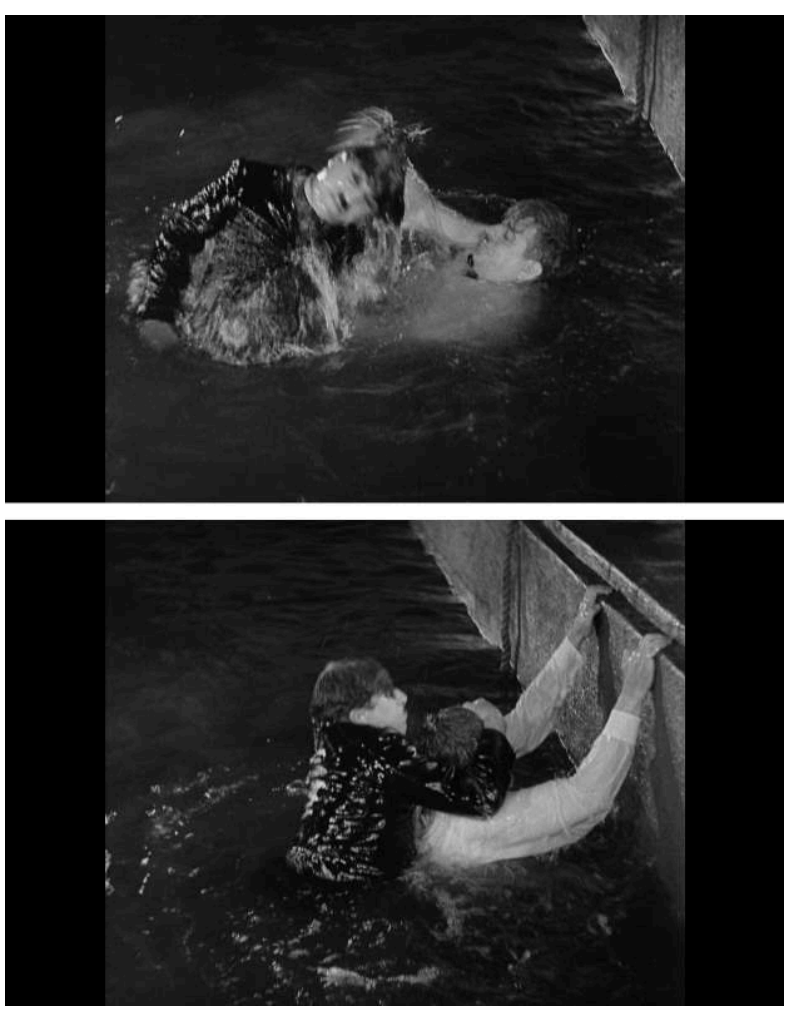

1 - Le mélodrame se noie dans la comédie (13'58)

2 - Le burlesque prend le dessus (14'53)

Charles Chaplin [Domaine public], DVD Les Lumières de la ville, MK2 Éditions, 2008.

24 La séquence suivante a lieu dans l'appartement du millionnaire, théâtre de l'affrontement entre deux univers dramatiques. Chacun des deux personnages poursuit la défense de son scénario: mélodramatique pour l'un, burlesque pour l'autre. Le millionnaire a procédé à l'enlèvement du clown et l'a entraîné dans un décor de mélodrame : le salon bourgeois dont deux éléments métaphoriques reflètent l'univers, le canapé et le piano. C'est justement à ces deux éléments caractéristiques que le clown va se heurter. Charlot, de plus en plus ivre, salit le canapé et se cogne contre le piano. 
Fig. 7. Extrait de City Lights (1931)

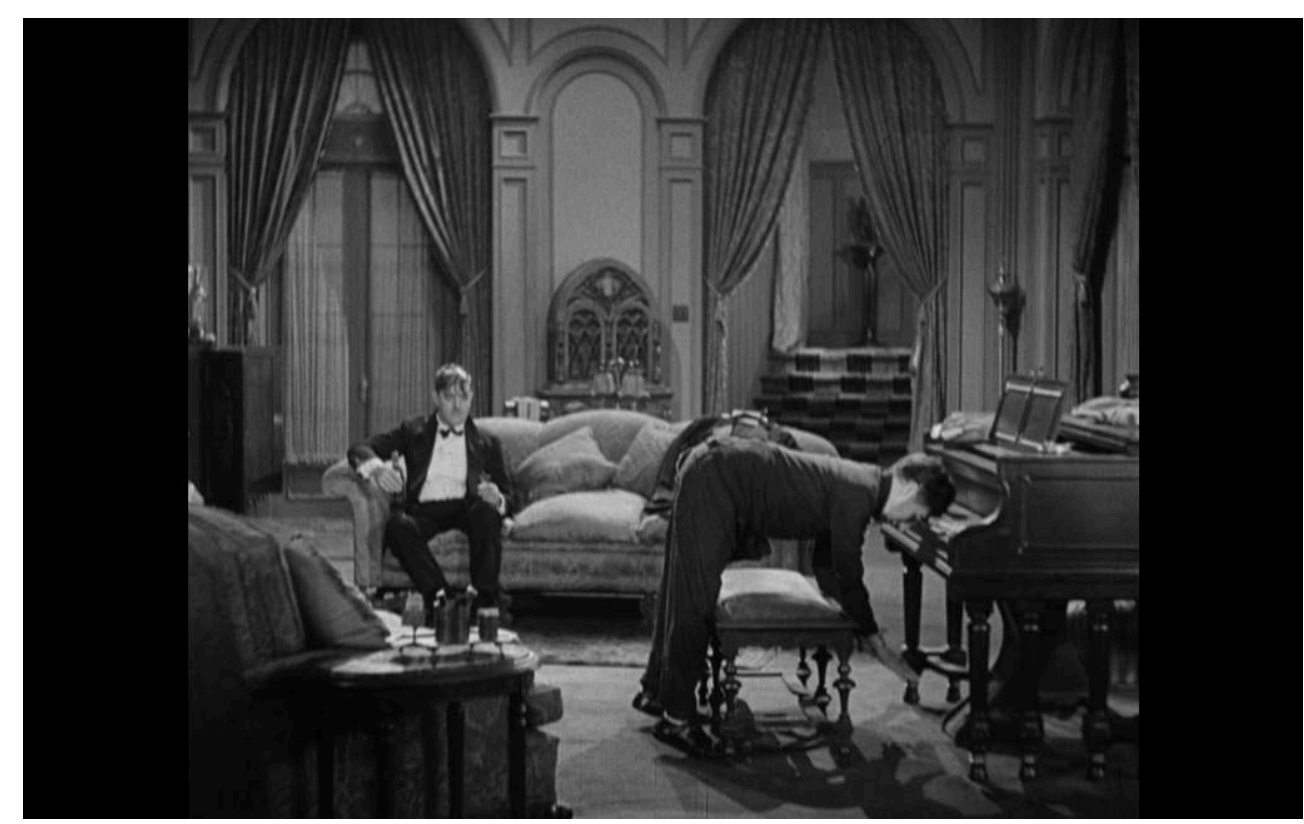

Charlot bute sur le décor du drame bourgeois (19'17)

Charles Chaplin [Domaine public], DVD Les Lumières de la ville, MK2 Éditions, 2008.

Le millionnaire a entrepris de s'enivrer et d'enivrer son nouveau compagnon. Cependant, l'alcool ne fait qu'augmenter ses "crises", accès intenses de sentiment mélodramatique : il se prend la tête entre les mains en signe de profond désespoir et sort un revolver. Il tient absolument à ce que l'histoire finisse mal. Charlot, lui, lutte pour la comédie et cette bataille héroïque contre le mélodrame est longue et ardue. Chaplin acteur continue à pousser la gestuelle du mélodrame jusqu'à épuisement de celui-ci et émergence du clown burlesque. Quand Charlot s'enivre, il est l'homme le plus ivre du monde, il s'engage avec ardeur dans la traversée de l'expérience. C'est l'un des traits fondamentaux de l'art du clown. Il faut imaginer que l'Auguste ${ }^{17}$ a vu depuis la coulisse ou le dernier rang du poulailler jouer le grand comédien des genres " sérieux », tels la tragédie, le drame ou le mélodrame. Il a tout absorbé, mais comme un spectateur étranger qui ne comprendrait pas la langue: les effondrements, les gestes de désespoir, les cris, les malédictions et les déclarations d'amour. Puis, Monsieur Loyal ${ }^{18}$, grand ordonnateur, l'a poussé en scène. Le clown doit convaincre le public qu'il est un grand acteur de drame puisque dans la hiérarchie - de convention du théâtre, la tragédie et le drame sont pris bien davantage au sérieux que la comédie. Il se précipite alors corps et âme dans une reconstitution de ce qu'il a vu, comme on parle de reconstitution d'une scène de crime. Et ce qu'il a retenu par-dessus tout, c'est la gravité de la situation. Tout est grave et c'est bien ainsi que le clown joue, en oubliant que tous les efforts qu'il déploie pour raconter le drame avec sérieux sont d'emblée anéantis par son masque qui dénonce son identité, par son innocence qui le pousse à l'excès, par sa maladresse qui produit la chute. Dans le cas de Charlot, son masque donne à voir, amplifiée, sa nature profondément inadaptée. C'est un vagabond, un étranger, un éternel immigré, c'est un lapsus vivant. Ainsi, sans le vouloir, en introduisant le chaos dans le décor et la fable du mélodrame, il en dénonce les ressorts et les valeurs.

Et Chaplin de montrer le cinéma du théâtre. 
l'une des premières scènes du film, lorsque Charlot rencontre dans la rue la jeune vendeuse de fleurs, il ne comprend pas tout de suite qu'elle est aveugle. Quand il s'en aperçoit, il est envahi d'émotion et l'on sait - puisqu'il déploie toute la panoplie du jeune homme amoureux - qu'il est sous le charme. Le vagabond ne tombe amoureux que de figures féminines tout droit sorties de l'univers du mélodrame: une orpheline dans Les Temps modernes (Modern Times), une immigrée sans le sou dans L'Émigrant (The Immigrant), une jeune artiste battue et affamée par son père dans Le Cirque (The Circus) et la jeune et pauvre aveugle de City Lights.

Alors que la vendeuse de fleurs le croit parti, Charlot, comme ensorcelé, revient doucement sur ses pas, s'assoit et l'observe, transi d'amour. Elle se lève, passe devant lui pour aller à la fontaine remettre de l'eau dans son vase. Elle verse l'eau, remue le vase et jette l'eau... sur Charlot qui perd tout à coup son masque de jeune premier. Ce rôle lui est décidément interdit. Le clown reprend alors sa gestuelle burlesque, celle d'un pantin désarticulé. La jeune aveugle, elle, ne se rend compte de rien. Le mélodrame est aveugle et ignore la comédie. 
Fig. 8. Extraits de City Lights (1931)
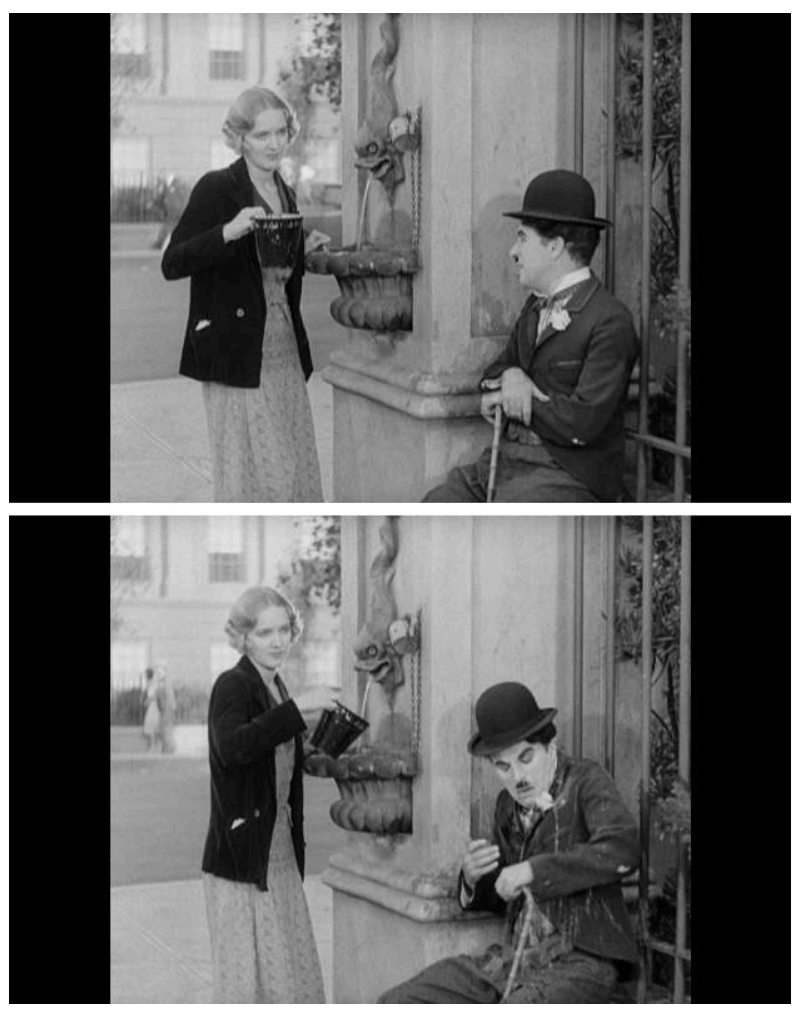

1 - Le mélodrame est aveugle (9'55)

2 - II ignore la comédie (9'56)

Charles Chaplin [Domaine public], DVD Les Lumières de la ville, MK2 Éditions, 2008.

Dans une autre séquence qui fonctionne comme un écho à cette rencontre entre les deux héros du film, Charlot raccompagne la jeune fille chez elle. Il agit en tout point comme un jeune premier. Il dévore des yeux l'objet de sa flamme, voyant sans être vu, ce qui lui donne toute liberté de s'épancher. Ne pouvant se résoudre à se priver de la contempler, il décide de la regarder par la fenêtre de sa maison. Il grimpe sur un tonneau et peut ainsi découvrir le lieu où elle vit. Il s'agit, encore une fois, d'un décor typique de mélodrame. Charlot est fasciné, mais un accident survient à nouveau, il tombe en renversant le tonneau plein d'eau. Chaplin inonde le décor du mélodrame. L'eau, si présente dans ce film, est la métaphore du geste politique autant qu'esthétique du cinéaste : le mélodrame se retrouve inondé, emporté par la vague du burlesque, les larmes sont noyées dans le rire. 
Fig. 9. Extraits de City Lights (1931)
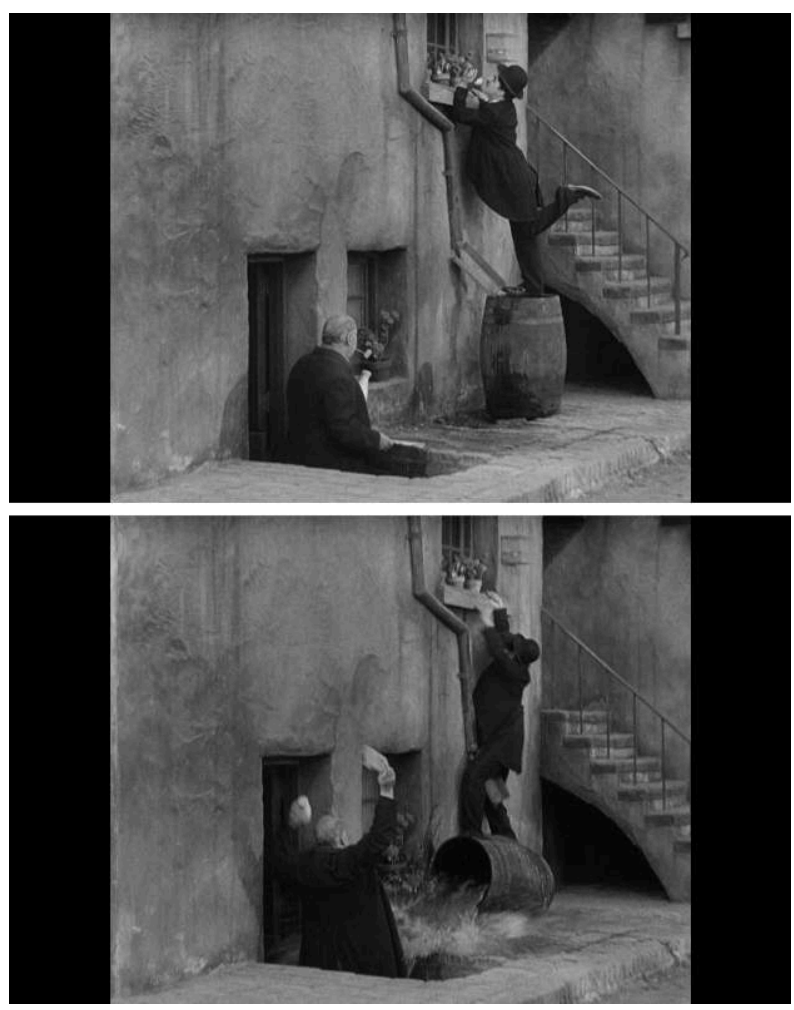

1 - Charlot observe sa belle (33'59)

2 - Et Chaplin inonde le mélodrame (34'04)

Charles Chaplin [Domaine public], DVD Les Lumières de la ville, MK2 Éditions, 2008.

Dans l'espace offert par l'écran, nous assistons à la chorégraphie d'un corps entré en résistance, celui de l'acteur Chaplin, cet "acteur anti-acteur", selon la formule de Jacques Rancière (2011: 241). En résistance contre l'illusion et l'identification, propres au système de représentation traditionnel. En résistance ${ }^{20}$ également contre la puissance de l'intrigue mélodramatique. La lutte entre les deux systèmes expressifs, celui de la mimesis naturaliste et celui de l'aveu de la diegesis, se traduit dans une mécanique de jeu qui fait naître une émotion universelle. Et cette mécanique ne fonctionne que parce qu'elle s'enraye :

La belle machine ne marche que par ses ratés. Le petit homme à la canne et au chapeau melon n'est pas plus un sentimental caché sous l'allure d'un automate qu'il n'est un biomécanicien masqué sous les dehors d'un benêt de comédie. Sa gestique de virtuose de la maladresse qui rate tout ce qu'il réussit et réussit tout ce qu'il rate fait de lui un habitant exemplaire d'un nouvel univers sensible. (Rancière 2011 : 241)

Car, contrairement à ce que pensaient certains avant-gardistes, inquiets de voir leur idole tomber dans la sentimentalité et confondant ainsi récit de Chaplin et fable de Charlot, confondant aussi mécanique parfaite et perfection de l'art, l'art surgit dans les failles de cette mécanique.

Le conte que nous donne à voir Chaplin se dédouble ainsi à plusieurs niveaux. Le cinéma cite et détourne les canevas du mélodrame et du drame bourgeois. Il parodie 
aussi le jeu mélodramatique, celui qui avait cours encore sur les scènes et que certains réalisateurs comme Griffith - pour n'en citer qu'un seul - ont importé au cinéma. Ce faisant, il met à mort toute illusion de la représentation. Quand la comédie burlesque vient bousculer la fable mélodramatique, quand l'acteur exhibe - à travers l'art du clown - avec une précision mécanique, un personnage noyé dans la fable, le cinéaste nous convie à l'agilité critique et transforme sous nos yeux l'anecdotique en symbolique. Alors que le jeu psychologique propre au drame bourgeois fait du personnage un cas particulier et ne donne aucune possibilité au public de s'échapper de la suffocation émotionnelle pour aller vers une réflexion politique, le jeu masqué de Chaplin joue avec les codes en faisant mine de les respecter, y introduit l'excès et en démonte la mécanique. En cela, Chaplin ne pouvait que devenir un modèle pour tous ceux qui cherchaient à faire progresser l'art de l'acteur et à le soustraire à un servile assujettissement au sens prédéfini inclus dans des fables faites sur mesure pour endormir le spectateur sous la domination d'une idéologie.

En lui, se fondent le créateur et la création, car, comme l'indique Louis Delluc :

[...] il est son peintre propre. Il est l'œuvre et l'auteur à la fois. Il a fait cette chose qui n'est possible qu'au cinéma - le dandysme m'excuse - c'est-à-dire: peindre, modeler, sculpter à même sa propre chair et son visage, une transposition d'art. (Delluc $1985: 84$ )

\section{BIBLIOGRAPHIE}

Barthes, Roland, 1957, Mythologies, Paris, Seuil, coll. « Points ».

-, [1973] 2002, « Diderot, Brecht, Eisenstein », dans CEuvres complètes, t. IV (livres, textes, entretiens, 1972-1976), Paris, Seuil.

Brecht, Bertolt, 1972, Écrits sur le théâtre, Paris, L'Arche.

Bordat, Francis, 1991, « Les engagements d'un artiste », dans Alain Masson (dir.), Hollywood 1927-1941, la propagande par les rêves ou le triomphe du modèle américain, Revue Autrement, Série Mémoires nº 9, Paris, Autrement, p. 127-135.

Chaplin, Charles, 1978, Histoire de ma vie, trad. Jean Rosenthal, Paris, Robert Laffont.

Damour, Christophe, 2009, «Les enseignants de la Méthode aux États-Unis », dans Fabien Gaffez (dir.), L'Actors Studio, une révolution stylistique ?, Amiens, Festival international du film d'Amiens, p. 71-85.

Damour, Christophe, 2007, «L'influence de Delsarte sur le jeu de l'acteur de cinéma aux ÉtatsUnis », dans Vincent Amiel, Jacqueline Nacache, Geneviève Sellier et Christian Viviani (dir.), L'acteur de cinéma : approches plurielles, Rennes, Presses Universitaires de Rennes, coll. « Le Spectaculaire ", p. 19-31.

Delluc, Louis, [1921] 1985, « Charlot », dans Écrits cinématographiques, t. I, Le Cinéma et les Cinéastes, Paris, La Cinémathèque française, p. 13-30.

Lang, Robert, 2008, Le Mélodrame américain (Griffith, Vidor, Minnelli), Paris, L’Harmattan. 
Meyerhold, Vsevolod, 1973, Écrits sur le théâtre, t. I, trad., préface et notes de Béatrice PiconVallin, Lausanne, L'Âge d'Homme, coll. « Théâtre $\mathrm{xx}^{\mathrm{e}}$ siècle ».

Naremore, James, 1988, Acting in the Cinema, Berkeley, Los Angeles, Londres, University of California Press.

Nysenholc, Adolphe, 2004, «Chaplin et Brecht », Brecht à Bruxelles, Alternatives théâtrales, $\mathrm{n}^{\circ} 81$, p. 80-83.

Picon-Vallin, Béatrice, 1990, « Le cinéma, rival, partenaire ou instrument du théâtre meyerholdien? ", dans Claudine Amiard-Chevrel (dir.), Théâtre et cinéma années vingt, t. I, textes réunis et présentés par Claudine Amiard-Chevrel, Lausanne, L'Âge d'Homme, coll. « Théâtre années vingt », p. 229-262.

Rancière, Jacques, 2011, Aisthesis, Scènes du régime esthétique de l'art, Paris, Galilée.

Rémy, Tristan, 2002, Les Clowns, Paris, Grasset.

Robinson, David, 1995, Charlot, entre rire et larmes, trad. Frédéric Maurin, Paris, Gallimard, coll. « Découvertes ».

Stanislavski, Konstantin, 2006, La Construction du personnage, trad. Charles Antonetti, Paris, Pygmalion.

\section{NOTES}

1. Pour saisir l'importance du mélodrame dès les origines du cinéma, voir l'ouvrage de Robert Lang (2008).

2. L'expression "jeu masqué» s'utilise le plus souvent parmi les gens de théâtre pour caractériser une interprétation qui utilise le port d'un masque. Cependant, certains pédagogues et artistes se réfèrent à une approche de l'interprétation dans laquelle, même si l'acteur ne porte pas de masque, persiste la trace des règles que ce dernier impose. Cette école de jeu puise donc son langage et sa codification dans l'art du masque, bien qu'il n'en fasse plus usage. C'est dans ce dernier sens que nous employons cette expression.

3. Nom anglo-saxon, et donc original, de Charlot.

4. Ces derniers forment, concernant l'art de l'acteur, le socle des avant-gardes théâtrales. Leur influence se propagera dans le champ du cinéma.

5. Nous entendons par avant-gardes théâtrales les tendances artistiques qui, en marquant leur claire opposition au théâtre dominant, insufflent à la pratique du théâtre de nouveaux codes esthétiques et éthiques.

6. Deux penseurs ont particulièrement attiré l'attention sur cette notion de théâtralité authentique : Meyerhold et Vakhtangov. Meyerhold en fait le fondement de son théâtre de la convention: voir son article de 1907, «Histoire et technique du théâtre » (Meyerhold 1973: 81-117).

7. Tous les dessins et croquis que Charlot a inspirés aux artistes du Bauhaus comme à ceux du constructivisme et du cubisme le prouvent.

8. On cite souvent Buster Keaton et son visage sans expression comme exemple de neutralité et de refus de psychologisme. Si l'art de Keaton évoque celui du masque neutre, le jeu de Chaplin évoque celui des masques expressifs, tout aussi dénué de psychologisme. En effet, les émotions qui le traversent sont enracinées dans l'archaïsme universel. Le jeu psychologiste, lui, utilise une panoplie de procédés saisis dans le lexique conventionnel des sentiments repérables.

9. L'influence de Chaplin se remarque aussi jusque dans l'écriture des pièces. Adolphe Nysenholc en a relevé la trace dans Maître Puntila et son valet Matti. Dans cette pièce de 1940, Puntila n'est 
généreux que quand il est ivre, comme le personnage du milliardaire dans City Lights (Nysenholc $2004: 83)$.

10. Stanislavski, pourtant considéré comme le père du jeu psychologique, est un des premiers théoriciens à considérer le personnage comme un masque : «[...] le personnage est le masque qui dissimule l'individu-acteur. Ainsi protégé, l'acteur peut dénuder son âme jusqu'au détail le plus intime. C'est là un des points les plus importants de la construction du personnage » (Stanislavski $2006: 48$ ).

11. Au milieu du XVIII ${ }^{\mathrm{e}}$ siècle, Denis Diderot expose dans les préfaces de ses pièces et dans ses ouvrages de réflexion une conception de l'art dramatique appelée à marquer de son empreinte le théâtre occidental. Il conçoit et défend l'idée d'une scène-miroir, où personnages, situations et décors reflètent la réalité sociale de son époque. Au XIX siècle, tandis que la bourgeoisie s'installe au pouvoir, on assiste à une véritable percée de ce mouvement dans les pièces représentées sur les scènes des boulevards. Mélodrames, drames bourgeois, vaudevilles, comédies de mœurs genres fraîchement éclos - développent des thèmes puisés dans le quotidien bien plus que ne le faisaient la tragédie ou la comédie du Grand Siècle. Vers 1850, ces genres forment le théâtre officiel, c'est-à-dire celui qui reçoit les faveurs du public comme des critiques. Ceux qui prêchent pour une refonte totale de l'art dramatique, les avant-gardistes - et notamment les symbolistes nomment ce théâtre dominant «théâtre bourgeois ». Ils reprochent à ce dernier d'être le défenseur du réalisme, un terme qui, pour eux, désigne la négation même de toute démarche artistique.

12. Il s'agit de la traduction littérale de Verfremdungseffekt.

13. Nous nous permettons d'utiliser cette expression qui évoque l'époque médiévale, mais nous faisons bien référence à la codification des transports amoureux du XIX ${ }^{\mathrm{e}}$ siècle.

14. Après avoir observé les comportements humains selon une démarche de nature scientifique, François Delsarte crée une typologie des postures en écho à une grammaire des sentiments. Il cherche ainsi à établir un catalogue des gestes spontanés et de leur lien avec une émotion spécifique. Cette méthode a eu un énorme retentissement aux États-Unis (bien plus qu'en France) où les disciples de Delsarte ont formé des générations de danseurs et de comédiens. Cependant Delsarte n'a pas échappé à la caricature hâtive et sa réputation a longtemps souffert d'extrapolations réductrices, telle la diffusion massive de dessins de gammes d'expression élevés au rang de manuels de catéchisme appliqué. Delsarte clamait haut et fort qu'il ne fallait surtout pas se limiter à imiter les postures, qu'elles devaient seulement servir d'indication et qu'en aucun cas elles ne représentaient une fin en soi. Voir en ce qui concerne les déviations de la méthode Delsarte l'article de Christophe Damour (2007 : 19-31).

15. «[...] c'est un vagabond qui agit follement comme un amant».

16. «[...] l'exagération comique de postures théâtrales démodées ».

17. Auguste est le nom que l'on donne traditionnellement au clown de cirque portant un nez rouge. Il apparaît souvent sur la piste en duo avec le clown blanc. Ce dernier, digne et autoritaire, représente le respect de l'ordre. Il est le faire-valoir de l'Auguste, qui, lui, introduit le chaos. À ce sujet, voir Tristan Rémy (2002).

18. Dans l'univers du cirque, Monsieur Loyal est le maître de la piste. C'est lui qui orchestre les différents numéros et particulièrement les entrées des clowns. Il exerce sur ceux-ci son pouvoir autoritaire.

19. On dit dans le langage du cirque et de l'art des masques que l'Auguste se met en enfer luimême. Il est en effet la première victime du chaos qu'il provoque malgré lui (malgré le personnage et non malgré l'acteur) sur son passage.

20. Selon Francis Bordat (1991 : 132), City Lights est également un film qui entre en résistance contre le cinéma sonore. 


\section{RÉSUMÉS}

Charlie Chaplin fait œuvre originale lorsque, en équilibre entre burlesque et pathétique, il convie devant sa caméra, pour la plus cathartique des rencontres, les canevas du mélodrame et la figure du clown. Celui-ci est appliqué, d'une absolue bonne foi, et cet excès d'application crée un hiatus entre ce qu'il s'attache à reproduire et sa propre identité, décalée et précaire. Lorsque Charlot, le clown, surgit dans le décor et la fable du mélodrame en y introduisant le chaos, Chaplin le cinéaste en dénonce les ressorts et les valeurs. La comédie burlesque vient bousculer la fable mélodramatique et, tandis que l'acteur exhibe avec une précision mécanique, un personnage noyé dans la fable, le cinéaste nous convie à l'agilité critique, nous met en garde contre les mécanismes des fables bourgeoises et transforme sous nos yeux l'anecdotique en symbolique.

Charlie Chaplin's work presents itself in his original form when, balancing between burlesque and pathos and for the most cathartic meeting, he invites both the framework of melodrama and the clown figure in front of his camera. The clown is hardworking, with profound good faith and being overdiligent breeds a hiatus between what he strives to copy and its own off-the-wall and unstable identity. When The Tramp suddenly bursts out on the scenery and amidst the fable of the melodrama bringing with him chaos, Chaplin the moviemaker denounces its patterns and its values. Then the burlesque comedy shakes up the melodramatic fable and when, via the art of the clown, the actor exhibits a character lost in the fable with mechanical accuracy, the moviemaker invites us to quick criticism, warns us against the mechanisms of bourgeois, conventional, fables while turning under our very eyes what's anecdotal into symbolism.

\section{INDEX}

Mots-clés : acteur, mélodrame, cirque, clown, parodie, jeu masqué

Keywords : actor, melodrama, circus, clown, parody, mask acting

\section{AUTEUR}

\section{ANNE POGGIOLI}

Docteur de l'université Paris 7 Diderot, chercheuse indépendante, comédienne 